\title{
Editor's note: From M2M to IoT: Platform, Network and Service
}

Published online: 15 August 2017

(C) Springer Science+Business Media, LLC 2017

Peer-to-Peer Networking and Applications gratefully acknowledges the editorial work of the scholars listed below on the special issue entitled "From M2M to IoT: Platform, Network and Service":

Young-June Choi, Ajou University, South Korea,

Ashwini Kumar, Arista Networks, USA

Zhetao Li, Xiangtan University, China

Hiroo Sekiya, Chiba University, Japan

The 8 papers in this issue include:

"OPPO: An optimal copy allocation scheme in mobile opportunistic networks" by Peiyan Yuan and Chenyang Wang

"Resource allocation for M2M-enabled cellular network using Nash bargaining game theory" by Gang Wang and Tanhui Liu

"A traffic congestion aware vehicle-to-vehicle communication framework based on Voronoi diagram and information granularity" by Gang Li, Bin He and Aimin Du
"Towards the oneM2M standards for building IoT ecosystem: Analysis, implementation and lessons" by Jaeho Kim, Sung-Chan Choi, Jaeseok Yun, JangWon Lee

"Distributed scheduling using belief propagation for internet-of-things (IoT) networks" by Illsoo Sohn, Sang Won Yoon, and Sang Hyun Lee

"CTMR-collaborative time-stamp based multicast routing for delay tolerant networks in post disaster scenario" by Amit Kumar Gupta, Jyotsna Kumar Mandal, Indrajit Bhattacharya, Tamal Mondal and Sourav Sanu Shaw

"Delay-tolerant sensing data delivery for IoT network by using signal strength information" by Sun-Hyun Kim and Seung-Jae Han

"Towards secure authenticating of cache in the reader for RFID-based IoT systems" by Chun-Ta Li, Cheng-Chi Lee, Chi-Yao Weng, and Chien-Ming Chen 